\title{
High energy factorization in nucleus-nucleus collisions III. Long range rapidity correlations
}

\author{
${\text { François } \operatorname{Gelis}^{(1,2)} \text {, Tuomas Lappi }}^{(2)}$, Raju Venugopalan ${ }^{(3)}$ \\ 1. Theory Division, PH-TH, Case C01600, CERN, \\ CH-1211, Geneva 23, Switzerland \\ 2. Institut de Physique Théorique (URA 2306 du CNRS) \\ CEA/DSM/Saclay, Bât. 774 \\ 91191, Gif-sur-Yvette Cedex, France \\ 3. Physics Department, Brookhaven National Laboratory \\ Upton, NY-11973, USA
}

\begin{abstract}
We obtain a novel result in QCD for long range rapidity correlations between gluons produced in the collision of saturated high energy hadrons or nuclei. This result, obtained in a high energy factorization framework, provides strong justification for the Glasma flux tube picture of coherent strong color fields. Our formalism can be applied to "near side ridge" events at RHIC and in future studies of long range rapidity correlations at the LHC.
\end{abstract}

\section{Introduction}

Long range rapidity correlations in high energy hadronic collisions are of interest in QCD because causality dictates that these correlations are produced at very early times. They therefore provide insight into how color correlations in the hadron wavefunctions become dynamically manifest in multiparticle final states. Recent observations in nucleus-nucleus collisions at RHIC of a "near side ridge" structure in two-particle correlations [1/2]3 and significant forwardbackward multiplicity correlations [4] have reinvigorated interest in the underlying dynamics of these correlations in QCD. At the LHC, with its wider rapidity coverage, such correlation studies can prove a powerful diagnostic tool both of multiparton correlations in QCD and of highly coherent strong color fields generated at early times in nuclear collisions.

Long range rapidity correlations were previously studied in color flux tube models where the non-perturbative dynamics is at the QCD scale $\Lambda_{\mathrm{QCD}} \sim 1$ 
$\mathrm{fm}^{-1}$. However, the rapid growth of parton distributions, and the requirement that occupation numbers in QCD saturate at $\sim 1 / \alpha_{\mathrm{s}}$, where $\alpha_{\mathrm{s}}$ is the QCD coupling constant, suggests that the dynamics of color correlations is controlled instead by a semi-hard saturation scale $Q_{s} \gg \Lambda_{\mathrm{QCD}}$. The properties of gluons with maximal occupation are described by the Color Glass Condensate (CGC) [5]; this saturated regime and the approach to it can be computed in a weak coupling effective field theory (EFT).

In the CGC EFT, partons with longitudinal momenta $k^{+}$larger than a cutoff momentum $\Lambda^{+}$in a high energy hadron or nucleus (moving in the $+z$ direction) are described as static light-cone color sources while modes with $k^{+}<\Lambda^{+}$are treated as QCD gauge fields that couple to these color sources [6]. Because the physics is independent of this separation scale, one obtains a renormalization group (RG) equation-the JIMWLK equation [7]-describing the evolution of the distribution of fast sources as $\Lambda^{+}$is lowered.

The QCD matter formed immediately after a nucleus-nucleus collision is a Glasma 8]. At leading order (LO), solutions of the Yang-Mills equations reveal that the Glasma corresponds at early times $\tau \lesssim Q_{s}^{-1}$ to highly coherent longitudinal chromoelectric and chromomagnetic field configurations 9]10] with maximal occupation numbers $1 / \alpha_{\mathrm{s}}$. At leading order, the Glasma fields are invariant under boosts in the space-time rapidity $\eta$ and only depend on their transverse positions in the nuclei and the proper time $\tau$. Further, the LO Glasma fields have the spatial structure of flux tubes stretching between the two nuclei, each localized transversely in a region of size $1 / Q_{s}$. This geometrical picture naturally explains the rapidity correlations observed in the near side ridge in heavy ion collisions [11/12]. However, the boost invariance of the Glasma configurations at $\mathrm{LO}$ is broken by quantum effects and it is important to understand their impact on multiparticle correlations.

In two previous papers 1314 -hereafter referred to as papers I and II- we applied this effective field theory to the inclusive multigluon spectra in nucleusnucleus collisions. The main result in these papers is a proof of the fact that all the leading logarithms that arise in loop corrections to these quantities can be absorbed into universal distributions for the fast sources of the two nuclei. However, in the case of the multigluon spectra, our proof was limited to the very peculiar situation where all the observed gluons lie in a small region in rapidity (of size $\Delta y \ll \alpha_{\mathrm{s}}{ }^{-1}$ ). This limitation was due to the fact that we did not resum corrections of the form $\alpha_{\mathrm{s}}\left|y_{i}-y_{j}\right|$ where $y_{i, j}$ are the rapidities of the gluons $i$ and $j$. These corrections become important when the rapidity separation between the observed gluons is of order $\alpha_{\mathrm{s}}{ }^{-1}$ or larger. Physically, these corrections arise from the radiation of extra gluons between those that are measured. This has a high probability of occurring if the rapidity interval between two measured gluons is larger than $\alpha_{\mathrm{s}}{ }^{-1}$.

The goal of the present paper, the third in this series, is to extend the treatment in the previous papers to compute inclusive multigluon spectra (to leading logarithmic accuracy) for arbitrary rapidity intervals between the observed gluons. In the case of the two-gluon spectrum, this is the basis for a detailed quan- 
titative study of long range rapidity correlations in heavy ion collision: 1 . These results are new and are valid (for any number of colors including the physical $N_{c}=3$ ) in a weak coupling scheme where higher order in $\alpha_{\mathrm{s}}$ contributionsenhanced by the same powers of the rapidity-are resummed to all orders. In this leading logarithmic approximation, we will demonstrate that expectation values of operators can be factorized as a convolution of density functionals from each of the nuclei times the operator computed with leading order classical fields. These density functionals evolve according to the JIMWLK equation and are universal; they can be extracted, for instance, in electron-nucleus collisions. Albeit the focus here will be on nucleus-nucleus collisions, the results also apply to the collision of two "saturated" hadrons at very high energies.

We note that while multiparticle correlations in the strong interactions have been extensively studied [15]16, none of the literature addresses nucleus-nucleus collisions, for finite $N_{c}$, in a framework where gluon fields are the dynamical degrees of freedom. We will comment later on interesting earlier studies [15] on two-particle correlations in the context of Local Reggeon Field Theory.

The essence of the "technology" needed to resum all the leading logs in multigluon spectra for arbitrary rapidity separations is already contained in papers I and II, albeit in a somewhat hidden form. In section 2, we review the main results of these papers and we prove a general formula for the renormalization group flow in the CGC when one moves the cutoff $\Lambda^{+}$of the effective theory by an infinitesimal amount. In section 3. we show how 1- and 2-gluon inclusive spectra can be obtained from this master formula. Our formula for the 2-gluon spectrum is expressed in terms of the usual distributions of color sources, and of a new object that has the interpretation of a propagator (in functional space) for the JIMWLK evolution. In the limit where the two gluons are nearby in rapidity, we recover known results for the 2-gluon spectrum. Finally, in section 4. we discuss the complications that arise when one wants to extend these results to the case where one (or both) of the projectiles is dilute instead of being in the saturation regime. We end with a brief summary and outlook for future work.

\section{Leading logarithms at NLO}

Consider an inclusive multigluon field operator $\mathcal{O}$. In papers I and II, we have shown that its LO value $\mathcal{O}_{\text {LO }}$ can be expressed in terms of light-cone gauge classical Yang-Mills solutions $\mathcal{A}^{\mu}$, with retarded boundary conditions $\lim _{x^{0} \rightarrow-\infty} \mathcal{A}^{\mu}(x)=0$. In coordinate space, in the CGC effective field theory, the classical sources are localized along the light-cones in two strips $0 \leq x^{-} \leq 1 / \Lambda^{+}$

\footnotetext{
${ }^{1}$ In [1112], a simpler leading order formula was used that does not resum the corrections in $\alpha_{\mathrm{s}}\left|y_{i}-y_{j}\right|$. This was sufficient to justify the existence of long range rapidity correlations and to suggest its relevance for the RHIC data. In particular, for STAR data, where the relevant rapidity window is $\Delta y \sim 1.5$, these resummation effects are not likely to be large. Resummations of long range rapidity corrections are however expected to improve the quantitative description of the STAR and PHOBOS data 2.3] and in future of the LHC data that has a significantly wider rapidity coverage.
} 


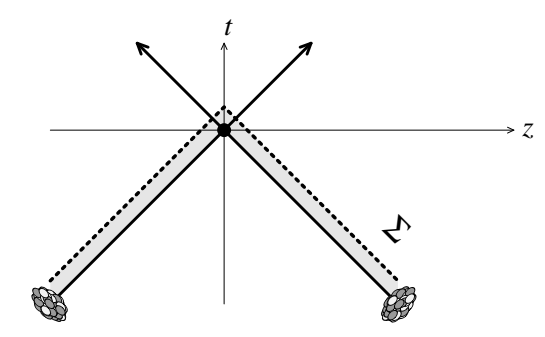

Figure 1: Initial space-time surface $\Sigma$ used in the initial value problem for the retarded classical field $\mathcal{A}^{\mu}$.

and $0 \leq x^{+} \leq 1 / \Lambda^{-}$. We denote by $\Sigma$ the surface located at a distance $\delta x^{ \pm}=1 / \Lambda^{\mp}$ above the backward light-cone, as illustrated in fig. 11. Because the classical fields involved in $\mathcal{O}_{\mathrm{LO}}$ obey retarded boundary conditions, $\mathcal{O}_{\mathrm{LO}}$ can be obtained by solving an initial value problem with initial conditions defined on the surface $\Sigma$.

It is convenient to describe color sources in the nuclei by distributions of Wilson lines

$$
\Omega_{1,2}\left(y, \boldsymbol{x}_{\perp}\right) \equiv \mathrm{T} \exp i g \int_{0}^{x_{y}^{\mp}} d z^{\mp} \frac{1}{\nabla_{\perp}^{2}} \widetilde{\rho}_{1,2}\left(z^{\mp}, \boldsymbol{x}_{\perp}\right)
$$

where $\widetilde{\rho}_{1,2}$ are the color source densities in Lorenz gauge. Here the upper bound $x_{y}^{\mp}$ in the integral is related to $y$ by $y \equiv \ln \left(P^{ \pm} x_{y}^{\mp}\right)$, with $P^{ \pm}$the longitudinal momenta of the respective nuclei 2 .

In papers I and II, the next-to-leading order (NLO) inclusive multigluon spectrum was shown to take the form

$$
\begin{aligned}
\mathcal{O}_{\mathrm{NLO}} & =\left\{\int_{\Sigma} \mathrm{d}^{3} \overrightarrow{\boldsymbol{u}}\left[\beta \cdot \mathbb{T}_{\boldsymbol{u}}\right]\right. \\
& \left.+\frac{1}{2} \sum_{\lambda, a} \int \frac{\mathrm{d}^{3} \boldsymbol{k}}{(2 \pi)^{3} 2 E_{\boldsymbol{k}}} \int_{\Sigma} \mathrm{d}^{3} \overrightarrow{\boldsymbol{u}} \mathrm{d}^{3} \overrightarrow{\boldsymbol{v}}\left[a_{-\boldsymbol{k} \lambda a} \cdot \mathbb{T}_{\boldsymbol{u}}\right]\left[a_{+\boldsymbol{k} \lambda a} \cdot \mathbb{T}_{\boldsymbol{v}}\right]\right\} \mathcal{O}_{\mathrm{LO}}
\end{aligned}
$$

up to terms that do not contribute at leading logarithmic accuracy. The fields $\beta^{\mu}(x)$ and $a_{ \pm \boldsymbol{k} \lambda a}^{\mu}(x)$ are small quantum fluctuations propagating over the classical field $\mathcal{A}^{\mu}$. The former has a vanishing boundary condition in the remote past and its evolution is driven by a source term consisting of a 1-loop tadpole graph. The latter evolves without any source term, but its boundary condition in the remote past is a plane wave $T^{a} \epsilon_{\lambda}^{\mu} e^{ \pm i k \cdot x}(\lambda, a$ and $\boldsymbol{k}$ denote the polarization, color and momentum of the initial fluctuation). Here, $d^{3} \overrightarrow{\boldsymbol{u}}$ is the measure on $\Sigma$.

\footnotetext{
${ }^{2}$ With this convention, $y$ is the rapidity measured from the beam rapidity and $y=0$ corresponds to including only valence partons that have $0 \leq x^{\mp} \leq 1 / P^{ \pm}$.
} 
Further, $\mathcal{O}_{\text {LO }}$ is a functional of the value of the classical field $\mathcal{A}^{\mu}$ on the surface $\Sigma$ and the differential operator $\mathbb{T}_{\boldsymbol{u}}$ acting on $\mathcal{O}_{\mathrm{LO}}$ is the generator of shifts of the value of $\mathcal{A}^{\mu}$ on $\Sigma$. The only part of the expression for this operator that is important for computing leading logarithmic contributions is 3

$$
a \cdot \mathbb{T}_{\boldsymbol{u}}=\partial_{\mu}\left(\Omega_{1,2}^{a b} a_{b}^{\mu}\right) \frac{\delta}{\delta \partial_{\mu}\left(\Omega_{1,2}^{a c} \mathcal{A}_{c}^{\mu}(u)\right)},
$$

with $\Omega_{1,2}$ defined as in eq. (11) with $y \equiv \ln \left(P^{ \pm} / \Lambda^{ \pm}\right)$.

The next step in evaluating the NLO corrections is to integrate over the momentum $\boldsymbol{k}$ in eq. (2). One integral appears explicitly in the term that has two operators $\mathbb{T}$, and another momentum integral is hidden in the source term of the fluctuation field $\beta^{\mu}$. Since in the CGC effective theory the modes described as fields are bounded by $k^{ \pm}<\Lambda^{ \pm}$, these longitudinal momentum integrals have an upper bound. We shall compute only the contribution of modes in the small slices $\Lambda^{\prime+}<k^{+}<\Lambda^{+}$and $\Lambda^{\prime-}<k^{-}<\Lambda^{-}$. By integrating out the field modes in these slices, one is going from the original CGC EFT to a new $\mathrm{CGC}^{\prime} \mathrm{EFT}$. The latter differs from the former because it has an additional layer of (slower) sources while its field modes now extend only up to smaller cutoffs $\Lambda^{\prime \pm}$.

Using the results of paper I, we have to leading logarithmic accuracy,

$$
\begin{gathered}
\int_{\Sigma} \mathrm{d}^{3} \overrightarrow{\boldsymbol{u}}\left[\beta \cdot \mathbb{T}_{\boldsymbol{u}}\right]+\frac{1}{2} \sum_{\lambda, a} \int \frac{\mathrm{d}^{3} \boldsymbol{k}}{(2 \pi)^{3} 2 E_{\boldsymbol{k}}} \int_{\Sigma} \mathrm{d}^{3} \overrightarrow{\boldsymbol{u}} \mathrm{d}^{3} \overrightarrow{\boldsymbol{v}}\left[a_{-\boldsymbol{k} \lambda a} \cdot \mathbb{T}_{\boldsymbol{u}}\right]\left[a_{+\boldsymbol{k} \lambda a} \cdot \mathbb{T}_{\boldsymbol{v}}\right] \\
\Lambda^{ \pm \pm<k^{ \pm}<\Lambda^{ \pm}} \ln \left(\frac{\Lambda^{+}}{\Lambda^{\prime+}}\right) \mathcal{H}_{\Lambda^{+}}+\ln \left(\frac{\Lambda^{-}}{\Lambda^{\prime-}}\right) \mathcal{H}_{\Lambda^{-}}
\end{gathered}
$$

In this equation, $\mathcal{H}_{\Lambda^{ \pm}}$are the JIMWLK Hamiltonians of the right and left moving nuclei respectively, at the scales $\Lambda^{ \pm}$. For the nucleus moving in the $+z$ direction, the explicit form of the JIMWLK Hamiltonian is

$$
\mathcal{H}_{\Lambda^{+}} \equiv \frac{1}{2} \int_{\boldsymbol{x}_{\perp}, \boldsymbol{y}_{\perp}} \frac{\delta}{\delta \widetilde{\mathcal{A}}_{a}^{+}\left(\epsilon^{-}, \boldsymbol{y}_{\perp}\right)} \eta_{1}^{a b}\left(\boldsymbol{x}_{\perp}, \boldsymbol{y}_{\perp}\right) \frac{\delta}{\delta \widetilde{\mathcal{A}}_{b}^{+}\left(\epsilon^{-}, \boldsymbol{x}_{\perp}\right)},
$$

with $\epsilon^{-}=1 / \Lambda^{+}$and where 4

$$
\begin{aligned}
& \eta_{1}^{a b}\left(\boldsymbol{x}_{\perp}, \boldsymbol{y}_{\perp}\right)=\frac{1}{\pi} \int \frac{d^{2} \boldsymbol{u}_{\perp}}{(2 \pi)^{2}} \frac{\left(\boldsymbol{x}_{\perp}^{i}-\boldsymbol{u}_{\perp}^{i}\right)\left(\boldsymbol{y}_{\perp}^{i}-\boldsymbol{u}_{\perp}^{i}\right)}{\left(\boldsymbol{x}_{\perp}-\boldsymbol{u}_{\perp}\right)^{2}\left(\boldsymbol{y}_{\perp}-\boldsymbol{u}_{\perp}\right)^{2}} \\
& \quad \times\left[1+\Omega_{1}\left(\boldsymbol{x}_{\perp}\right) \Omega_{1}^{\dagger}\left(\boldsymbol{y}_{\perp}\right)-\Omega_{1}\left(\boldsymbol{x}_{\perp}\right) \Omega_{1}^{\dagger}\left(\boldsymbol{u}_{\perp}\right)-\Omega_{1}\left(\boldsymbol{u}_{\perp}\right) \Omega_{1}^{\dagger}\left(\boldsymbol{y}_{\perp}\right)\right]_{a b} .
\end{aligned}
$$

There is a similar definition for the second nucleus moving in the $-z$ direction.

\footnotetext{
${ }^{3}$ The complete operator $a \cdot \mathbb{T}_{\boldsymbol{u}}$ is made of three terms, but two of them (not written explicitly here) do not provide any leading logarithmic contributions.

${ }^{4}$ Wilson lines without a rapidity argument are defined as in eq. (1) with $y \equiv \ln \left(P^{ \pm} / \Lambda^{ \pm}\right)$ - they integrate over all the sources of the CGC EFT down to the cutoff $\Lambda^{ \pm}$. Moreover, the derivatives in the JIMWLK Hamiltonian are with respect to the slowest sources of the EFT.
} 
An important point to note here is that the relation in eq. (4) is a property of the operator enclosed in the curly brackets of eq. (2), regardless of the details of the observable $\mathcal{O}$ under consideration. The second remarkable property of this result is that the leading logarithms can be assigned to one or the other of the two nuclei, without any mixing that would violate factorization.

The expectation value of $\mathcal{O}$, at NLO, can be represented in the CGC effective theory as

$$
\begin{aligned}
\left\langle\mathcal{O}_{\mathrm{LO}}\right. & \left.+\mathcal{O}_{\mathrm{NLO}}\right\rangle=\int\left[D \Omega_{1}\left(y, \boldsymbol{x}_{\perp}\right) D \Omega_{2}\left(y, \boldsymbol{x}_{\perp}\right)\right] \\
& \times W_{\Lambda^{+}}\left[\Omega_{1}\left(y, \boldsymbol{x}_{\perp}\right)\right] W_{\Lambda^{-}}\left[\Omega_{2}\left(y, \boldsymbol{x}_{\perp}\right)\right]\left[\mathcal{O}_{\mathrm{LO}}+\mathcal{O}_{\mathrm{NLO}}\right],
\end{aligned}
$$

where $W_{\Lambda^{ \pm}}\left[\Omega_{1,2}\left(y, \boldsymbol{x}_{\perp}\right)\right]$ are the functional probability distributions for the Wilson line configurations $\Omega_{1,2}\left(y, \boldsymbol{x}_{\perp}\right)$.

Inserting eq. (44) in eq. (21) and substituting the resulting expression on the r.h.s. of eq. (7), one can perform an integration by parts 5 such that $\mathcal{H}_{\Lambda^{ \pm}}$ operates on the distributions $W_{\Lambda^{ \pm}}$. Let us denote6

$$
W_{\Lambda^{\prime}+}\left[\Omega_{1}^{\prime}\left(y, \boldsymbol{x}_{\perp}\right)\right] \equiv[1+\underbrace{\ln \left(\frac{\Lambda^{+}}{\Lambda^{\prime+}}\right)}_{\mathrm{d} y} \mathcal{H}_{\Lambda^{+}}] W_{\Lambda^{+}}\left[\Omega_{1}\left(y, \boldsymbol{x}_{\perp}\right)\right] .
$$

This equation is the infinitesimal form of the JIMWLK evolution equation, where $\Omega_{1}^{\prime}$ is the Wilson line corresponding to a source distribution $\widetilde{\rho}_{1}^{\prime}=\widetilde{\rho}_{1}+\delta \widetilde{\rho}_{1}$ and $\delta \widetilde{\rho}_{1}$ has support only in the interval $[y, y+d y]$.

The argument of $W_{\Lambda^{\prime}+}$ in eq. (8) extends one step further in rapidity than the argument of $W_{\Lambda^{+}}$- it is defined over the range $0 \leq y \leq \ln \left(P^{+} / \Lambda^{\prime+}\right)$ and is hence a Wilson line in the $\mathrm{CGC}^{\prime} \mathrm{EFT}$. Thus our derivation proves that

$$
\langle\mathcal{O}_{\mathrm{LO}}+\underbrace{\mathcal{O}_{\mathrm{NLO}}}_{\Lambda^{ \pm \pm}<k^{ \pm}<\Lambda^{ \pm}}\rangle=\left\langle\mathcal{O}_{\mathrm{LO}}\right\rangle^{\prime}
$$

The prime on the r.h.s. indicates that the average is performed with a probability distribution corresponding to the Wilson lines of the CGC' effective theory. In other words, this identity states that the classical expectation value of $\mathcal{O}$ in the original EFT, corrected by quantum fluctuations in a small slice of field modes, can be expressed as a purely classical expectation value in a new EFT with a lower cutoff and with a distribution of Wilson lines evolved according to eq. (8).

Equation (9) describes how to resum the leading logarithmic quantum corrections in a small slice of longitudinal momentum. Successive leading logarithmic contributions down to $k^{ \pm}=0$ are obtained by repeating this elementary step infinitely many times while letting the thickness of the slices go to zero. One

\footnotetext{
${ }^{5}$ The JIMWLK Hamiltonian is Hermitian.

${ }^{6}$ An identical analysis also applies to the second nucleus.
} 
then obtains

$$
\langle\mathcal{O}\rangle_{\mathrm{LLog}}=\int\left[D \Omega_{1}\left(y, \boldsymbol{x}_{\perp}\right) D \Omega_{2}\left(y, \boldsymbol{x}_{\perp}\right)\right] W\left[\Omega_{1}\left(y, \boldsymbol{x}_{\perp}\right)\right] W\left[\Omega_{2}\left(y, \boldsymbol{x}_{\perp}\right)\right] \mathcal{O}_{\mathrm{LO}},
$$

where $W \equiv \lim _{\Lambda^{ \pm} \rightarrow 0} W_{\Lambda^{ \pm}}$. This expression is the central result of this paper. Eq. (10) shows that all the leading logarithms of rapidity, whether they correspond to the rapidity intervals between the nuclei and the tagged gluons or between the various tagged gluons, can be absorbed into the probability distributions $W$ for the trajectories of Wilson lines of the two projectiles. This formula applies to any inclusive observable for which eq. (2) is valid, regardless of whether the observable is local in rapidity or not. Because our result contains an average over $y$-dependent "trajectories" of Wilson lines, rather than an average over Wilson lines at a given fixed rapidity, it contains a lot of information about multigluon correlations at different rapidities.

\section{One- and two-gluon inclusive spectra}

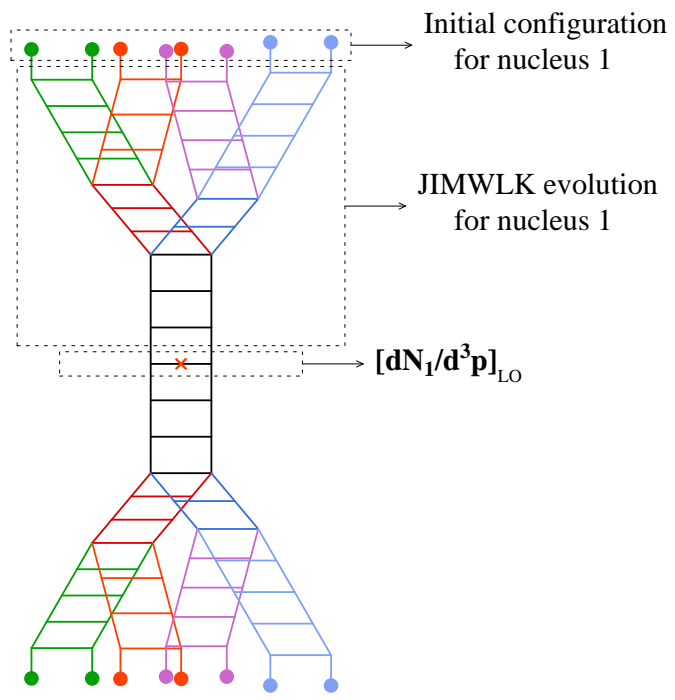

Figure 2: Diagrammatic representation of the various building blocks in the factorized formula for the inclusive single gluon spectrum. The lower part of the figure, representing nucleus 2 , is made up of identical building blocks.

We will now extract from our general result in eq. (10), expressions for single and double inclusive gluon spectra. The single inclusive gluon spectrum $d N_{1} / \mathrm{d}^{3} \boldsymbol{p}$ at LO depends only on the Wilson lines $\Omega_{1,2}\left(y, \boldsymbol{x}_{\perp}\right)$ at the rapidity $y=y_{p}$ of the produced gluon and not on the whole rapidity range as in eq. (10). 
Therefore, we can simplify eq. (10) by inserting the identity

$$
1=\int\left[D U_{1,2}\left(\boldsymbol{x}_{\perp}\right)\right] \delta\left[U_{1,2}\left(\boldsymbol{x}_{\perp}\right)-\Omega_{1,2}\left(y_{p}, \boldsymbol{x}_{\perp}\right)\right]
$$

and by defining the corresponding probability distributions for configurations of Wilson lines at the rapidity $y_{p}$

$Z_{y_{p}}\left[U_{1,2}\left(\boldsymbol{x}_{\perp}\right)\right] \equiv \int\left[D \Omega_{1,2}\left(y, \boldsymbol{x}_{\perp}\right)\right] W\left[\Omega_{1,2}\left(y, \boldsymbol{x}_{\perp}\right)\right] \delta\left[U_{1,2}\left(\boldsymbol{x}_{\perp}\right)-\Omega_{1,2}\left(y_{p}, \boldsymbol{x}_{\perp}\right)\right]$.

One then obtains the all order leading log result for the single inclusive gluon spectrum in the following form

$$
\left.\left\langle\frac{\mathrm{d} N_{1}}{\mathrm{~d}^{2} \boldsymbol{p}_{\perp} d y_{p}}\right\rangle\right|_{\mathrm{LLog}}=\left.\int\left[D U_{1}\left(\boldsymbol{x}_{\perp}\right) D U_{2}\left(\boldsymbol{x}_{\perp}\right)\right] Z_{y_{p}}\left[U_{1}\right] Z_{y_{p}}\left[U_{2}\right] \frac{\mathrm{d} N_{1}\left[U_{1}, U_{2}\right]}{\mathrm{d}^{2} \boldsymbol{p}_{\perp} \mathrm{d} y_{p}}\right|_{\mathrm{LO}} .
$$

Note that the distribution $Z_{y_{p}}[U]$ obeys the JIMWLK equation,

$$
\partial_{y_{p}} Z_{y_{p}}[U]=\mathcal{H}_{y_{p}} Z_{y_{p}}[U]
$$

which must be supplemented by an initial condition at a rapidity close to the fragmentation region of the projectiles. Eq. (13) is illustrated in the figure 2 ,

At Leading Order, the inclusive two-gluon spectrum is simply the disconnected product of two single gluon spectra [14|17] each of which depends on Wilson lines at the rapidity of the corresponding gluon. Using eq. (11) in eq. (10) (now we need to insert four such delta functions), one obtains for the resummed two-gluon spectrum the expression

$$
\begin{aligned}
& \left.\frac{\mathrm{d} N_{2}}{\mathrm{~d}^{2} \boldsymbol{p}_{\perp} \mathrm{d} y_{p} \mathrm{~d}^{2} \boldsymbol{q}_{\perp} \mathrm{d} y_{q}}\right|_{\text {LLog }}=\int\left[D U_{1}^{p}\left(\boldsymbol{x}_{\perp}\right) D U_{2}^{p}\left(\boldsymbol{x}_{\perp}\right) D U_{1}^{q}\left(\boldsymbol{x}_{\perp}\right) D U_{2}^{q}\left(\boldsymbol{x}_{\perp}\right)\right] \\
& \times\left.\left. Z_{y_{p}, y_{q}}\left[U_{1}^{p}, U_{1}^{q}\right] Z_{y_{p}, y_{q}}\left[U_{2}^{p}, U_{2}^{q}\right] \frac{\mathrm{d} N_{1}\left[U_{1}^{p}, U_{2}^{p}\right]}{\mathrm{d}^{2} \boldsymbol{p}_{\perp} \mathrm{d} y_{p}}\right|_{\text {LO }} \frac{\mathrm{d} N_{1}\left[U_{1}^{q}, U_{2}^{q}\right]}{\mathrm{d}^{2} \boldsymbol{q}_{\perp} \mathrm{d} y_{q}}\right|_{\text {LO }},(15)
\end{aligned}
$$

where we have introduced the double probability distribution of Wilson lines as

$$
\begin{aligned}
& Z_{y_{p}, y_{q}}\left[U_{1,2}^{p}, U_{1,2}^{q}\right] \equiv \int\left[D \Omega_{1,2}\left(y, \boldsymbol{x}_{\perp}\right)\right] W\left[\Omega_{1,2}\left(y, \boldsymbol{x}_{\perp}\right)\right] \\
& \times \delta\left[U_{1,2}^{p}\left(\boldsymbol{x}_{\perp}\right)-\Omega_{1,2}\left(y_{p}, \boldsymbol{x}_{\perp}\right)\right] \delta\left[U_{1,2}^{q}\left(\boldsymbol{x}_{\perp}\right)-\Omega_{1,2}\left(y_{q}, \boldsymbol{x}_{\perp}\right)\right] .
\end{aligned}
$$

This double distribution obeys the JIMWLK equation with respect to the largest of the two rapidities,

$$
\text { if } y_{q}>y_{p}, \quad \partial_{y_{q}} Z_{y_{p}, y_{q}}\left[U^{p}, U^{q}\right]=\mathcal{H}_{y_{q}} Z_{y_{p}, y_{q}}\left[U^{p}, U^{q}\right],
$$

with the boundary condition

$$
\lim _{y_{q} \rightarrow y_{p}} Z_{y_{p}, y_{q}}\left[U^{p}, U^{q}\right]=Z_{y_{p}}\left[U^{p}\right] \delta\left[U^{p}-U^{q}\right] .
$$


Alternately, this double distribution can be expressed as

$$
Z_{y_{p}, y_{q}}\left[U^{p}, U^{q}\right]=G_{y_{q}, y_{p}}\left[U^{q}, U^{p}\right] Z_{y_{p}}\left[U^{p}\right],
$$

where the Green's function $G_{y_{q}, y_{p}}\left[U^{q}, U^{p}\right]$ satisfies the JIMWLK equation]

$$
\partial_{y_{q}} G_{y_{q}, y_{p}}\left[U^{q}, U^{p}\right]=\mathcal{H}_{y_{q}} G_{y_{q}, y_{p}}\left[U^{q}, U^{p}\right]
$$

with the initial condition

$$
\lim _{y_{q} \rightarrow y_{p}} G_{y_{q}, y_{p}}\left[U^{q}, U^{p}\right]=\delta\left[U^{q}-U^{q}\right] .
$$

This Green's function describes multigluon evolution, between two tagged gluons, in the presence of strong color sources from the projectiles.

Our result for the double inclusive gluon spectrum, to leading logarithmic accuracy, can thus be expressed as follows

$$
\begin{gathered}
\left.\left\langle\frac{\mathrm{d} N_{2}}{\mathrm{~d}^{2} \boldsymbol{p}_{\perp} \mathrm{d} y_{p} \mathrm{~d}^{2} \boldsymbol{q}_{\perp} \mathrm{d} y_{q}}\right\rangle\right|_{\text {LLog }}=\int\left[D U_{1}^{p}\left(\boldsymbol{x}_{\perp}\right) D U_{2}^{p}\left(\boldsymbol{x}_{\perp}\right) D U_{1}^{q}\left(\boldsymbol{x}_{\perp}\right) D U_{2}^{q}\left(\boldsymbol{x}_{\perp}\right)\right] \\
\times Z_{y_{p}}\left[U_{1}^{p}\right] G_{y_{p}, y_{q}}\left[U_{1}^{p}, U_{1}^{q}\right] Z_{y_{q}}\left[U_{2}^{q}\right] G_{y_{q}, y_{p}}\left[U_{2}^{q}, U_{2}^{p}\right] \\
\quad \times\left.\left.\frac{\mathrm{d} N_{1}\left[U_{1}^{p}, U_{2}^{p}\right]}{\mathrm{d}^{2} \boldsymbol{p}_{\perp} \mathrm{d} y_{p}}\right|_{\mathrm{LO}} \frac{\mathrm{d} N_{1}\left[U_{1}^{q}, U_{2}^{q}\right]}{\mathrm{d}^{2} \boldsymbol{q}_{\perp} \mathrm{d} y_{q}}\right|_{\text {LO }} .
\end{gathered}
$$

Eq. (22) generalizes the result in paper II - that result, as implied by eq. (21), is recovered when the rapidities of the two gluons are close to each other. Our formula for the two-gluon spectrum in eq. (22) is illustrated in fig. (3). By using eq. (10), it is straightforward to write down similar formulae for higher gluon correlations.

Factorization is obviously manifest in eq. (10). It is slightly less apparent in eq. (22) because there are more factors in the integrand. However, both the $Z$ functionals and the $G$ Green's functionals are universal objects that describe the partonic content of a nucleus at high energy. The part of the integrand which is specific to the observable under consideration is relegated to the factors $d N_{1} / d^{3} \boldsymbol{p}$ and $d N_{1} / d^{3} \boldsymbol{q}$.

Note also that these distributions are far more general than the $k_{\perp}$-dependent correlators of Wilson lines that are often discussed in the literature of high energy QCD. The latter appear in a form of factorization which goes under the rubric of $k_{\perp}$-factorization. This type of factorization is formulated in terms of single gluon distributions, but is known to be violated 16 for 2gluon correlations in collisions involving at least one saturated projectile. It is in this context that one should interpret the results of [15] which concludes that factorization is violated. In stark contrast, our universal density functionals and

\footnotetext{
${ }^{7}$ We use here, and previously, the fact that the JIMWLK Hamiltonian acts only on objects at equal or higher rapidities.
} 


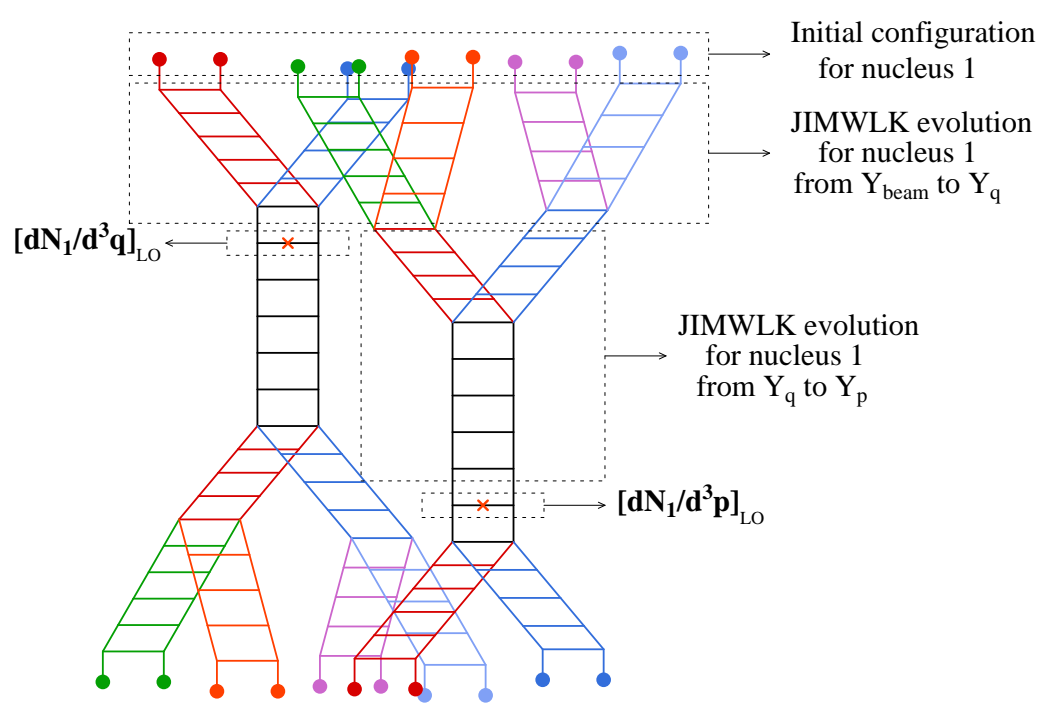

Figure 3: Diagrammatic representation of the various building blocks in the factorized formula for the inclusive 2-gluon spectrum. As in the previous figure, the corresponding evolution from nucleus 2 at the bottom of the figure is not shown explicitly.

Green's functions contain all the relevant information on rapidity dependent $n$ gluon correlations. In fact, our factorization result is a general consequence of causality and for this reason should even be valid beyond leading log accuracy.

In practice, solving the JIMWLK equation to compute the r.h.s of eq. (22) is more conveniently achieved by writing this equation as a Langevin equation for Wilson lines living on the $\mathrm{SU}(3)$ group manifold [18. This stochastic formulation was implemented in the only extant numerical study of the JIMWLK equation [19. Because solving the JIMWLK equation can be numerically challenging, a simpler formulation of high energy evolution is provided by the Balitsky-Kovchegov (BK) equation [20, which is a closed form mean field simplification of the JIMWLK expression for 2-point Wilson line correlators in the CGC 8. The BK equation corresponds to a nonlocal Gaussian form of the $Z_{y_{p}}, Z_{y_{q}}$ functionals 21] (when expressed in terms of the color source distributions) with a variance $\mu_{A}^{2}\left(y_{p, q}, x_{\perp}\right)$. Because the weight functionals at both $y_{p}$ and $y_{q}$ have this Gaussian form, the Green's function $G_{y_{p}, y_{q}}$ connecting the two must also be a Gaussian, whose variance can be determined from numerical solutions of the BK equation. Therefore, quantitative results for eq. (22) can be obtained within this BK framework. These will be discussed in future work.

${ }^{8}$ The BK equation is valid in the large $N_{\mathrm{c}}$ limit for large nuclei. 


\section{Dilute-dense limit}

The results obtained here are valid for the collision of two dense projectiles whose color charge densities of both are given by $\widetilde{\rho}_{1,2} \sim g^{-1}$. This is the case in large nuclei or in nucleons at very high energies. An interesting question is whether decreasing the magnitude of $\widetilde{\rho}_{1}$ and/or $\widetilde{\rho}_{2}$ in the formulas we have obtained so far gives the correct answer for dilute-dense or dilute-dilute collisions. Before going further, let us note that the permitted range for dilute to dense color charge densities is between $g$ and $g^{-1}$. The upper value, assumed in our study, corresponds to a fully saturated projectile. The lower value corresponds to a very dilute projectile whose parton density is of order unity ensuring that its density of color charge is proportional to $g$.

The answer to the question posed is affirmative for the single inclusive gluon spectrum (eq. (13)). By taking the limit $\widetilde{\rho}_{2} \sim g$ in this formula, one recovers immediately the well known result for the single inclusive spectrum in pA collisions and likewise for $p p$ collisions when we let both $\widetilde{\rho}_{1,2}$ become of order $g$.

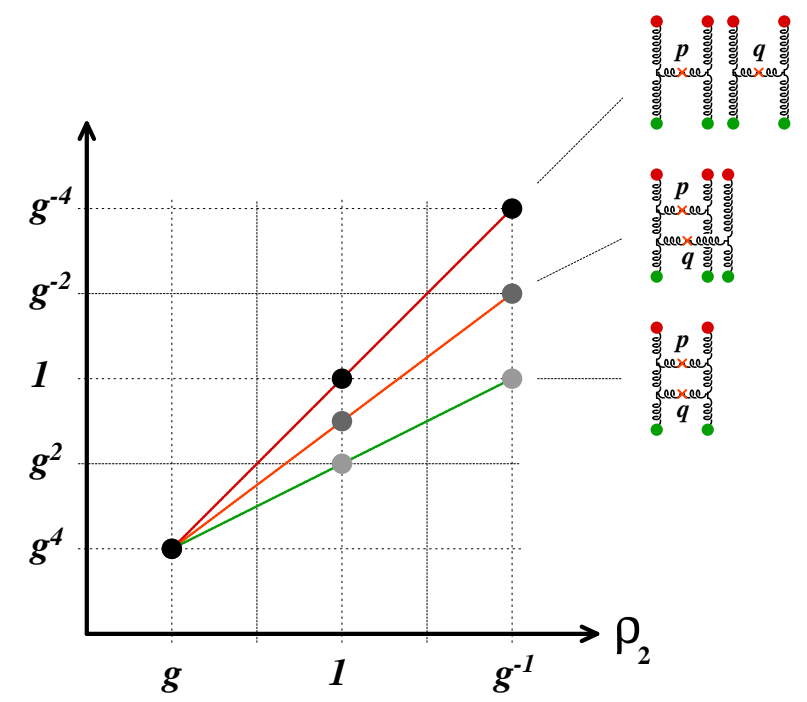

Figure 4: Order of magnitude of various contributions to the 2-gluon spectrum as a function of the color charge density $\rho_{2}$ in the small projectile (the color charge density in the large projectile is held fixed $\rho_{1}=\mathcal{O}\left(g^{-1}\right)$ ). Large logarithms of the energy, which become relevant in the leading logarithmic resummation, are not considered here.

However, taking this limit in the 2-gluon spectrum (eq. (22)) does not lead to the correct results for the inclusive 2-gluon spectrum in pA or pp collisions [16]. The reason of this discrepancy is that the corresponding power counting for the two gluon spectrum is very different for "dense" color sources $\sim g^{-1}$ relative to the case of "dilute" sources $\sim g$. In the power counting for dense sources 
$\sim g^{-1}$, certain graphs are suppressed that would also be leading graphs for dilute sources $\sim g$. This is illustrated in figure 4, where we have displayed the order of magnitude of three different contributions to the 2-gluon spectrum, as a function of $\widetilde{\rho}_{2}$. (For pA collisions, $\widetilde{\rho}_{1}$ is fixed to be of order $g^{-1}$.) One sees that in the dense case, only one of these graphs is important, while they are all important in the dilute limit. Since eq. (22) is obtained by assuming dense projectiles, it contains only the disconnected graph and misses the other two - this implies that eq. (22) is not the complete answer in the dilute limit. As noted, this subtlety affects correlations between two or more gluons but not the single gluon spectrum.

The previous discussion only deals with contributions at Leading Order. However, a similar discrepancy between the power counting in the dense-dense and dilute-dense limits occurs in the resummation of the leading logarithmic contributions. There, one sees that the operators included in the JIMWLK Hamiltonian are not the only ones that contribute at leading log accuracy in the dilute regime. For instance, when $\widetilde{\rho} \sim g$, an operator of the form $g^{4} \widetilde{\rho}^{2}(\delta / \delta \widetilde{\rho})^{4}$ has the same order of magnitude as the operators in the JIMWLK Hamiltonian in the dilute limit (but is totally suppressed in the dense regime). Such an operator, with a prefactor of order $\widetilde{\rho}^{2}$ and four derivatives with respect to the color source, corresponds to "pomeron splittings" in the energy evolution [16|22 23|24|25] - while the JIMWLK evolution only has "pomeron mergings" (because the number of $\widetilde{\rho}$ 's in the prefactor is always equal to or greater than the number of derivatives). In principle, one would like a formalism where both limits contain the right physics. Unlike previous works which address the full S-matrix for high energy scattering, our focus will be on the more limited goal of computing inclusive gluon spectra in dense-dilute collisions. We believe that substantial progress in this direction is feasible and will further address this topic in a future publication.

\section{Conclusions}

In this paper, we obtained in eq. (10) a general result for inclusive n-gluon production at arbitrary rapidities in the collision of two dense projectiles (such as heavy nuclei) with charge densities given by $\widetilde{\rho}_{1,2} \sim g^{-1}$. The result is expressed in terms of universal $W$-density matrix functionals which contain information on n-gluon correlations in the wavefunctions of the dense projectiles. Our formalism is strictly valid in the leading logarithmic approximation in $x$. We anticipate, on the basis of simple causality arguments, that the structure of our result will hold beyond leading logarithmic accuracy.

We explicitly wrote down the corresponding expressions for single and double inclusive gluon production with arbitrarily large rapidity separations between tagged gluons. Until this point, there was no microscopic QCD based formalism that allows the computation of the near side ridge correlations in nucleus-nucleus collisions when the rapidity separation between the measured particles is of the order of $1 / \alpha_{s}$ or more. Our formalism fills this gap and allows for future quanti- 
tative comparisons and predictions for the rapidity dependence of the the ridge like structures observed in central nucleus-nucleus collisions at RHIC and in future at the LHC. At the LHC, one may have the possibility of studying such structures that may span 6-10 units in rapidity. Such long range correlations therefore open a new window on the study of multiparton correlations in QCD as well as the provide a "chronometer" of the strong field initial "Glasma" stage of heavy ion collisions.

When both projectiles are dense, we argued that only "Pomeron mergings" that are fully included in the JIMWLK Hamiltonian are relevant for inclusive gluon production. When one or both of the projectiles becomes dilute, our power counting suggests that "Pomeron splitting" contributions, become equally important as the "merging" contributions for correlations involving two or more gluons. Because these are not included in the JIMWLK formalism, they cannot be obtained by taking a naive low density limit of the dense-dense formalism discussed in this paper. A smooth interpolation from the dilute-dense to the dense-dense limits for multigluon inclusive distributions requires that we first compute corrections to the JIMWLK Hamiltonian in the dilute-dense limit. While there have been several such studies in the context of the S-matrix for high energy scattering, they are in their infancy for inclusive multigluon production [26]. These studies will be important for extending our studies for nucleus-nucleus collisions to asymmetric systems such as high energy proton-

nucleus collisions. This work is in progress and will be addressed in a future publication.

\section{Acknowledgements}

We thank the Center for Theoretical Sciences of the Tata Institute for Fundamental Research for their support during the program "Initial Conditions in Heavy Ion Collisions". R.V.'s research is supported by the US Department of Energy under DOE Contract No. DE-AC02-98CH10886. F.G.'s work is supported in part by Agence Nationale de la Recherche via the programme ANR-06-BLAN-0285-01.

\section{References}

[1] J. Putschke, J. Phys. G34, S679 (2007).

[2] STAR, M. Daugherity, arXiv:0806.2121 [nucl-ex].

[3] PHOBOS, B. Alver et al., J. Phys. G35, 104080 (2008).

[4] STAR, B.K. Srivastava, Int. J. Mod. Phys. E16, 3371 (2007).

[5] E. Iancu and R. Venugopalan, arXiv:hep-ph/0303204 H. Weigert, Prog. Part. Nucl. Phys. 55, 461 (2005). 
[6] L. D. McLerran and R. Venugopalan, Phys. Rev. D49, 2233 (1994); ibid. 3352 (1994); ibid. D50, 2225 (1994).

[7] J. Jalilian-Marian, A. Kovner, L. D. McLerran and H. Weigert, Phys. Rev. D55, 5414 (1997); J. Jalilian-Marian, A. Kovner, A. Leonidov and H. Weigert, Nucl. Phys. B504, 415 (1997); ibid. Phys. Rev. D59, 014014 (1998); E. Iancu, A. Leonidov and L. D. McLerran, Nucl. Phys. A692, 583 (2001); ibid. Phys. Lett. B510, 133 (2001); E. Ferreiro, E. Iancu, A. Leonidov and L. McLerran, Nucl. Phys. A703, 489 (2002).

[8] T. Lappi and L. McLerran, Nucl. Phys. A772, 200 (2006).

[9] A. Kovner, L. D. McLerran and H. Weigert, Phys. Rev. D52, 3809 (1995).

[10] D. Kharzeev, A. Krasnitz and R. Venugopalan, Phys. Lett. B545, 298 (2002).

[11] A. Dumitru, F. Gelis, L. McLerran and R. Venugopalan, Nucl. Phys. A810, 91 (2008);

[12] S. Gavin, L. McLerran and G. Moschelli, arXiv:0806.4718 [nucl-th],

[13] F. Gelis, T. Lappi and R. Venugopalan, Phys. Rev. D78, 054019 (2008).

[14] F. Gelis, T. Lappi and R. Venugopalan, Phys. Rev. D78, 054020 (2008).

[15] M. Ciafaloni and G. Marchesini, Nucl. Phys. B109, 261 (1976); M.A. Braun, Eur. Phys. J. C48, 501 (2006); ibid. arXiv:0801.0493.

[16] J. Jalilian-Marian and Y. V. Kovchegov, Phys. Rev. D70, 114017 (2004).

[17] D. Kharzeev, E. Levin and L. McLerran, Nucl. Phys. A748, 627 (2005); N. Armesto, L. McLerran and C. Pajares, Nucl. Phys. A781, 201 (2007).

[18] J.-P. Blaizot, E. Iancu and H. Weigert, Nucl. Phys. A713, 441 (2003).

[19] K. Rummukainen and H. Weigert, Nucl. Phys. A739, 183 (2004).

[20] I. Balitsky, Nucl. Phys. B463, 99 (1996); Y. V. Kovchegov, Phys. Rev. D60, 034008 (1999).

[21] H. Fujii, F. Gelis and R. Venugopalan, Nucl. Phys. A780, 146 (2006).

[22] A. H. Mueller, A. I. Shoshi and S. M. H. Wong, Nucl. Phys. B 715 (2005) 440.

[23] E. Iancu and D. N. Triantafyllopoulos, Nucl. Phys. A 756 (2005) 419.

[24] A. Kovner and M. Lublinsky, Phys. Rev. D 71 (2005) 085004.

[25] A. Kovner and M. Lublinsky, Phys. Rev. D 72 (2005) 074023.

[26] A. Kovner and M. Lublinsky, JHEP 11, 083 (2006). 